\title{
Determination of the Baryon Density from Large Scale Galaxy Redshift Surveys
}

\author{
David M. Goldberg and Michael A. Strauss ${ }^{1}$ \\ Princeton University Observatory, Princeton, NJ 08544-1001
}

\begin{abstract}
We estimate the degree to which the baryon density, $\Omega_{b}$, can be determined from the galaxy power spectrum measured from large scale galaxy redshift surveys, and in particular, the Sloan Digital Sky Survey. A high baryon density will cause wiggles to appear in the power spectrum, which should be observable at the current epoch. We assume linear theory on scales $\geq 20 h^{-1} \mathrm{Mpc}$ and do not include the effects of redshift distortions, evolution, or biasing. With an optimum estimate of $P(k)$ to $k \sim 2 \pi /\left(20 h^{-1} \mathrm{Mpc}\right)$, the $1 \sigma$ uncertainties in $\Omega_{b}$ are roughly 0.07 and 0.016 in flat and open $\left(\Omega_{0}=0.3\right)$ cosmological models, respectively. This result suggests that it should be possible to test for consistency with big bang nucleosynthesis estimates of $\Omega_{b}$ if we live in an open universe.
\end{abstract}

Subject headings: methods: data analysis — methods: statistical — large scale structure of the universe - cosmology: theory

\section{Introduction}

In anticipation of the forthcoming Sloan Digital Sky Survey (hereafter SDSS; see http://www.astro.princeton.edu/BB00K; Gunn \& Weinberg 1995; Strauss 1997), several papers have discussed the use of large redshift surveys to determine of the galaxy power spectrum (Vogeley \& Szalay 1996; Tegmark et al. 1997ab; Hamilton 1997ab) and parameters derived therefrom (Tegmark 1997; Nakamura, Matsubara, \& Suto 1997; de Laix \& Starkman 1997). This paper will quantify how well we can constrain the baryon density, $\Omega_{b}$, from the power spectrum of the SDSS redshift survey.

\footnotetext{
${ }^{1}$ Alfred P. Sloan Foundation Fellow
} 
The universal ratio of baryonic mass density to critical mass density, $\Omega_{b}$, is of acute cosmological interest. In the standard big bang model, the ratio of baryon density to photon density, $\eta$, uniquely determines the relative abundances of deuterium, ${ }^{3} \mathrm{He},{ }^{4} \mathrm{He}$ and ${ }^{7} \mathrm{Li}$. Although the number density of photons can be readily measured from the temperature and spectrum of the Cosmic Microwave Background Radiation, the measurement of baryon density is much more difficult. However, by determining the abundance of deuterium, for example, one can compute a unique value of $\eta$ (Peebles 1993). From $\eta$ and the measured photon density one can determine $\Omega_{b}$, up to uncertainties in the Hubble constant, $H_{0}$. However, since deuterium is converted into ${ }^{3} \mathrm{He}$ in stars, it is necessary to look at very old objects in order to get an accurate estimate of the primordial deuterium abundance. Tytler, Fan, \& Burles (1996) measured the deuterium abundances in high redshift QSO absorption lines and determined $\Omega_{b}=0.024 \pm 0.002 h^{-2}$, where $h=H_{0} /\left(100 \mathrm{~km} \mathrm{~s}^{-1} \mathrm{Mpc}^{-1}\right)$. We shall adopt a value of $h=0.65$ (Kundić et al. 1997) throughout this paper, yielding a value of $\Omega_{b}=0.057 \pm 0.005$ from the Tytler et al. (1996) observations.

Can the baryon density be independently confirmed? The baryonic mass density can have a profound effect on the shape of the expected mass power spectrum on large scales. The baryonic component of the density field is strongly coupled to the radiation field until $z \simeq 1100$. There are fluctuations in the radiation power spectrum due to sound waves crossing the horizon at decoupling (Padmanabhan 1995; Hu \& Sugiyama 1996). Immediately after decoupling, the baryon power spectrum is the same as that of the photons, containing fluctuations, or "wiggles" on large scales. If the matter field is almost entirely composed of Cold Dark Matter (CDM), however, the baryons quickly fall into the dark matter potential wells, and much of these wiggles are erased. However, the greater the proportion of baryons, $\Omega_{b} / \Omega_{0}$, the greater the influence of the wiggles in the final (linearly evolving) power spectrum.

The change in shape of the power spectrum through decoupling has been modeled analytically by several authors. $T(k)$ is defined as the transfer function of the power spectrum such that $P(k) \propto k^{n_{\text {prim }}} T(k)^{2}$, where $n_{\text {prim }}$ is the spectral index of the primordial power spectrum. Holtzman (1989) computed $T(k)$ for a wide variety of models, including some with non-zero values of $\Omega_{b}$. Sugiyama (1995; see also White et al. 1996; Peacock \& Dodds 1994) include a baryon correction term to the analytic fit to the transfer function:

$$
T(k)=\frac{\ln (1+2.34 q)}{2.34 q} \times\left[1+3.89 q+(16.1 q)^{2}+(5.46 q)^{3}+(6.71 q)^{4}\right]^{-1 / 4} .
$$

Here, $q \equiv k / h \Gamma, \Omega_{0}$ is the ratio of mass density to the critical density, and

$$
\Gamma=\Omega_{0} h \exp \left[-\Omega_{b}-\left(\frac{h}{0.5}\right)^{1 / 2} \frac{\Omega_{b}}{\Omega_{0}}\right]
$$


This functional form is smooth; it does not contain the wiggles discussed above and thus this analytic formulation gives results which are entirely degenerate for various values of $\Omega_{b}$ and $\Omega_{0}$. If we take the wiggles into account, the degeneracy can be broken.

We use a numerical approach to quantify the effect of wiggles in the power spectrum. We use a package called CMBfast, written by Seljak \& Zaldarraiga (1996) to calculate the transfer function of the mass power spectrum for a wide variety of cosmological models.

Figure 1 illustrates the differences between the analytic fit of equation (11) and the numerical approach. In Figure 11a, we plot four power spectra, each with a value of $\Gamma=0.58$, and a normalization on large scales based on the COBE four year results (Bunn \& White 1997). Each assumes a value of $h=0.65$, and for comparison we plot both the numerical model and analytical fit with $\Omega_{0}=1, \Omega_{b}=0.057$, the numerical result for $\Omega_{0}=0.89$, $\Omega_{b}=0$, and the numerical result for $\Omega_{0}=1.37, \Omega_{b}=0.26$, which has the same value of $\Omega_{b} / \Omega_{0}$ as that found in the open model in panel b. Note that the $\Omega_{b}=0$ numerical model and the analytical fit are almost indistinguishable. This is because the two have identical values of $\Gamma$ and neither breaks this degeneracy with wiggles. Panel b shows an analytic fit and a numerical model of an $\Omega_{0}=0.3, \Omega_{b}=0.057$ power spectrum. Panel c shows the ratio of the analytic fit to the numerical model power spectrum for the flat, $\Omega_{b}=0.057$ case. Finally, panel d shows the same relationship for the open case. It is clear from panels c and $\mathrm{d}$ that the wiggles, while present in the flat universe model, have a much greater effect in an open universe. We propose to estimate how well $\Omega_{b}$ can be determined by examining these features. The emphasis in this paper is not to present a method of measuring $P(k)$, but to estimate errors on quantities on which $P(k)$ depends, especially $\Omega_{b}$.

The outline of the paper is as follows. In $\S$ we discuss the use of the Fisher information matrix in determining parameter uncertainties, and in $\S$ s we present the details of our method. $\S$ t contains the results of that method for several different cosmological models. In $\S$, we discuss other complicating factors which enter into the analysis of redshift survey power spectra. Finally, in $\S$ 6, we present our conclusions. In Appendix A, we present an alternate expansion of the SDSS density field, which may be useful for future computational work.

\section{How Well Can We Determine Parameters?}

Suppose that we are able to parameterize a power spectrum at the current epoch according to some set of parameters, $\Theta$. A moderately complete set might include the following parameters: $\Theta=\left\{\sigma_{8}, \Omega_{0}, \Omega_{b}, h, n_{\text {prim }}\right\}$, where the latter four have been defined 
previously, and $\sigma_{8}$ is a normalization constant, the rms density fluctuations within $8 h^{-1} \mathrm{Mpc}$ spheres. We assume that other parameters, such as $\Lambda$, are known exactly throughout this discussion; we set $\Lambda=0$.

In addition to a model, we have some data, $d_{i}, i=1, \ldots, n$ put into a data vector, $\mathbf{d}$, where $n$ is the total number of data elements. Moreover, we have a method to relate the raw observables (e.g. positions and redshifts of galaxies) to $\mathbf{d}$. In order to compare $\mathbf{d}$ to the model, we can associate some likelihood function $L(\boldsymbol{\Theta} ; \mathbf{d})$, which we would like to maximize in order to find the "true" cosmological parameters, $\boldsymbol{\Theta}_{0}$, where

$$
L=\frac{e^{-\mathbf{d}^{\dagger} \mathbf{C}^{-1} \mathbf{d} / 2}}{(2 \pi)^{N / 2} \operatorname{det}(\mathbf{C})^{1 / 2}} .
$$

This form assumes that $\left\langle d_{i}\right\rangle=0$ and that the expected distribution of $\mathbf{d}$ is a multivariate Gaussian.

The covariance matrix, $\mathbf{C}$, is defined as

$$
C_{l m} \equiv\left\langle d_{l} d_{m}^{*}\right\rangle .
$$

Following Tegmark, Taylor \& Heavens (1997, hereafter TTH), we use the Fisher information matrix to determine the minimum possible uncertainties in these parameters. The Fisher matrix is defined as

$$
F_{i j} \equiv\left\langle\frac{\partial^{2} \mathcal{L}}{\partial \theta_{i} \partial \theta_{j}}\right\rangle,
$$

where $\mathcal{L}=-\ln L$. This may further be reduced to (Vogeley \& Szalay 1996; TTH):

$$
F_{i j}=\frac{1}{2} \operatorname{Tr}\left[\mathbf{A}_{i} \mathbf{A}_{j}\right],
$$

where the matrices $\mathbf{A}_{i} \equiv \mathbf{C}^{-1} \partial \mathbf{C} / \partial \theta_{i}$.

The Fisher matrix allows us to determine the minimum possible errors for each parameter in a reasonable way via the Cramèr-Rao inequality (see TTH for a discussion). Regardless of how the actual parameters are measured, one cannot hope to do better than:

$$
\Delta \theta_{i} \geq\left(\mathbf{F}^{-1}\right)_{i i}^{1 / 2} .
$$

In the limit where all parameters but the $i^{\text {th }}$ are known with certainty, this becomes $\Delta \theta_{i} \geq 1 / \sqrt{F_{i i}}$. 


\section{Method}

In this section, we discuss how we define our data $\mathbf{d}$, and determine its covariance matrix, $\mathbf{C}(\S$ 3.1). Our aim is to use equation (6) to determine the Fisher information matrix $\mathbf{F}$ for the SDSS redshift survey power spectrum, and thus put an upper limit on the expected error in $\Omega_{b}$ via equation (7).

The SDSS northern redshift survey will cover an elliptical cone of $\pi$ steradians to an approximate depth of $600 h^{-1} \mathrm{Mpc}(z \simeq 0.2)$, with redshifts for approximately $10^{6}$ galaxies.

D. Weinberg (private communication) has created a mock SDSS redshift sample based on an $N$-body simulation by C. Park and J. R. Gott. We have used this mock survey to calculate a selection function:

$$
\bar{n}(r)=\frac{3}{\omega} \sum_{i, g a l} \frac{1}{d_{\max , i}^{3}},
$$

where $\omega$ is the solid angle of the survey and $d_{\text {max }, i}$ is the maximum distance that galaxy $i$ could be placed and still pass the selection criteria of the survey. The sum is carried out only over those galaxies for which $d_{\max , i}>r$. The selection function for the SDSS is plotted in Figure 2. We assume that the selection function has no angular dependence.

Our primary limitations in the calculation of $\mathbf{F}$ involve the computation, storage, and inversion of the covariance matrix, C. All three of these considerations will be immensely simplified if we can make $\mathbf{C}$ as close to diagonal as possible. We reduce covariance by an appropriate choice for the data $\mathbf{d}(\S 3.1)$ and with a clever choice of weighting $(\S 3.2)$. For the SDSS geometry, we find that we can indeed approximate the covariance matrix as diagonal for scales smaller than $1 / 8$ the largest dimension of the survey $(\S 3.4,3.5)$.

\subsection{Computing the Covariance Matrix}

We must first specify the form of the dataset, $\mathbf{d}$, that we will use. The method we use can be described as a "poor man's" Karhunen-Loève (K-L) transform. The K-L transform (Vogeley \& Szalay 1996; TTH) is a method of extracting the statistically independent elements of a dataset that provide the maximum ratio of signal to noise and hence most strongly constrain the cosmological parameters. Emulating the K-L transforms, we wish to minimize the values of the off-diagonal elements of the covariance matrix, since we will ultimately need to invert it (equation 3). If the survey volume were a cube with a uniform selection function, the Fourier modes of the density field with wavelengths along each axis given by integral fractions of the cube length, would indeed have no covariance for non-identical modes; they would be the K-L modes. We thus embed our survey volume in 
a cube of length $L$, which is specified by the largest dimension of the survey:

$$
L=2 r_{\max } \sin \theta_{\max }
$$

where $r_{\max }=809 h^{-1} \mathrm{Mpc}$ is chosen where $\bar{n}(r)$ first goes to zero, and $\theta_{\max }=1.13 \mathrm{rad}$ is the largest off-axis angle of the survey. This gives $L=1466 h^{-1} \mathrm{Mpc}$.

We use as our dataset $\mathbf{d}$ the Fourier transform of the real space density field

$$
\mathbf{k}\left(n_{x}, n_{y}, n_{z}\right)=\frac{2 \pi n_{x}}{L} \hat{\mathbf{x}}+\frac{2 \pi n_{y}}{L} \hat{\mathbf{y}}+\frac{2 \pi n_{z}}{L} \hat{\mathbf{z}}
$$

where $n_{x}, n_{y}$ and $n_{z}$ are non-negative integers. Because the SDSS geometry is of course not cubic, this scheme will not exactly diagonalize the covariance matrix, but we will see in $\S 3.5$ that we will be able to make the approximation that it is diagonal at large $|k|$. We order the modes in concentric cubes in $\mathbf{k}$ space, such that they are roughly ordered in increasing value of $|k|^{3}$. We refer to $\mathbf{k}_{l}$ as the $l^{\text {th }}$ value of $\mathbf{k}$ in this ordering. Thus there is a rough correspondence between increasing $l$ and decreasing scale. As the $\Omega_{b}$-induced wiggles are prominent on large scales, we will determine our Fisher matrix, and corresponding uncertainties on parameters, as a function of $n$, the maximum value of $l$ which we probe.

This is clearly not the only basis set which will partially diagonalize the covariance matrix from the outset. In Appendix A we discuss a set of basis vectors constructed from spherical Bessel functions and the spherical harmonics which will produce very little covariance within the SDSS geometry.

Following Fisher et al. (1993), we estimate the density field of galaxies as a sum of discrete points:

$$
\delta(\mathbf{r})=\sum_{i, g a l} \frac{\delta^{D}\left(\mathbf{r}-\mathbf{r}_{i}\right)}{\bar{n}(\mathbf{r})}-1,
$$

where $\bar{n}(\mathbf{r})$ is the selection function, the expected density of observed galaxies at $\mathbf{r}$ in the absence of clustering. We allow ourselves a weighting function, $\psi(\mathbf{r})$, in order to further reduce covariance and to minimize shot noise (see $\S 3.2$ ). The Fourier Transform of the density field, $\hat{\delta}(\mathbf{k})$, is then taken. Our Fourier convention here and throughout is:

$$
\hat{f}(\mathbf{k})=\int d^{3} \mathbf{r} e^{i \mathbf{k} \cdot \mathbf{r}} f(\mathbf{r})
$$

and

$$
f(\mathbf{r})=\frac{1}{(2 \pi)^{3}} \int d^{3} \mathbf{k} e^{-i \mathbf{k} \cdot \mathbf{r}} \hat{f}(\mathbf{k}) .
$$

\footnotetext{
${ }^{2}$ We discuss redshift-space distortions in $\S 5.2$.

${ }^{3}$ Note that unlike the K-L modes, we have not ordered the $d_{i}$ in decreasing signal-to-noise ratio.
} 
The Fourier modes of the weighted density are then:

$$
\hat{\delta}(\mathbf{k})=\int d^{3} \mathbf{r} e^{i \mathbf{k} \cdot \mathbf{r}} \psi(\mathbf{r}) \delta(\mathbf{r})
$$

Thus $\mathbf{d}$ is the set of $\hat{\delta}\left(\mathbf{k}_{l}\right)$, for the ordering of $\mathbf{k}_{l}$ described above. Substituting equation (11) gives:

$$
\hat{\delta}(\mathbf{k})=\sum_{i} \frac{\psi\left(\mathbf{r}_{i}\right) e^{i \mathbf{k} \cdot \mathbf{r}_{i}}}{\bar{n}\left(\mathbf{r}_{i}\right)}-W(\mathbf{k})
$$

where the window function, $W(\mathbf{k})$, is defined as:

$$
W(\mathbf{k}) \equiv \int d^{3} \mathbf{r} \psi(\mathbf{r}) e^{i \mathbf{k} \cdot \mathbf{r}}
$$

Taking the product of any two of these modes, we can compute the covariance:

$$
\begin{array}{r}
\hat{\delta}\left(\mathbf{k}_{1}\right) \hat{\delta}\left(\mathbf{k}_{2}\right)^{*}=\left(\sum_{i} \frac{\psi\left(\mathbf{r}_{i}\right)}{\bar{n}\left(\mathbf{r}_{i}\right)} e^{i \mathbf{k}_{1} \cdot \mathbf{r}_{i}}\right)\left(\sum_{j} \frac{\psi\left(\mathbf{r}_{j}\right)}{\bar{n}\left(\mathbf{r}_{j}\right)} e^{-i \mathbf{k}_{2} \cdot \mathbf{r}_{j}}\right) \\
-W\left(\mathbf{k}_{1}\right) \sum_{i} \frac{\psi\left(\mathbf{r}_{i}\right)}{\bar{n}\left(\mathbf{r}_{i}\right)} e^{-i \mathbf{k}_{2} \cdot \mathbf{r}_{i}}-W\left(\mathbf{k}_{2}\right)^{*} \sum_{i} \frac{\psi\left(\mathbf{r}_{i}\right)}{\bar{n}\left(\mathbf{r}_{i}\right)} e^{i \mathbf{k}_{1} \cdot \mathbf{r}_{i}}+W\left(\mathbf{k}_{1}\right) W\left(\mathbf{k}_{2}\right)^{*} .
\end{array}
$$

Following Fisher et al. (1993) we take the expectation value of this expression and after some algebra recover the expression:

$$
\left\langle\hat{\delta}\left(\mathbf{k}_{1}\right) \hat{\delta}\left(\mathbf{k}_{2}\right)^{*}\right\rangle=\frac{1}{(2 \pi)^{3}} \int d^{3} \mathbf{k}^{\prime} P\left(\mathbf{k}^{\prime}\right) W\left(\mathbf{k}_{1}-\mathbf{k}^{\prime}\right) W\left(\mathbf{k}_{2}-\mathbf{k}^{\prime}\right)^{*}+\int d^{3} \mathbf{r} \frac{\psi(\mathbf{r})^{2} e^{i\left(\mathbf{k}_{1}-\mathbf{k}_{2}\right) \cdot \mathbf{r}}}{\bar{n}(\mathbf{r})} .
$$

If the weighting function is normalized such that $\int d^{3} \mathbf{r} \psi(\mathbf{r})=1$, the diagonal elements of the covariance matrix are:

$$
C_{l l}=\tilde{P}\left(\mathbf{k}_{l}\right)+P_{s h o t}
$$

where

$$
\tilde{P}\left(\mathbf{k}_{l}\right)=\frac{1}{(2 \pi)^{3}} \int d^{3} \mathbf{k}^{\prime} P\left(\mathbf{k}^{\prime}\right)\left|W\left(\mathbf{k}_{l}-\mathbf{k}^{\prime}\right)\right|^{2}
$$

and

$$
P_{\text {shot }}=\int d^{3} \mathbf{r} \frac{\psi(\mathbf{r})^{2}}{\bar{n}(\mathbf{r})}
$$

which matches the result expected from traditional methods of power spectrum estimation (Fisher et al. 1993; Feldman, Kaiser, \& Peacock 1994, hereafter FKP; Park et al. 1994). 


\subsection{The Optimal Choice for $\psi(\mathbf{r})$}

We now discuss how the weighting function, $\psi(\mathbf{r})$, is chosen. First, the weighting function must reflect the survey geometry. That is, it must go to zero outside the survey and be well-defined within it. It must also minimize shot noise by giving regions with higher $\bar{n}(\mathbf{r})$ a higher weight. Finally, we wish to minimize covariance between modes. Tegmark (1995) gives a clever solution which minimizes covariance in an arbitrary geometry, and we shall use his algorithm throughout. The weighting function is the solution to the Schrödinger's Equation:

$$
\left[-\frac{1}{2} \nabla^{2}+\frac{\gamma}{\bar{n}(\mathbf{r})}\right] \psi(\mathbf{r})=E \psi(\mathbf{r}),
$$

where $E$ is the smallest eigenvalue of the system, and $\gamma$ is a constant with units of length ${ }^{-5}$ which balances the desire to minimize covariance while maximizing the signal to noise ratio. We used $\gamma=10^{-8} h^{5} \mathrm{Mpc}^{-5} \approx n_{\text {gal }} L^{-5}$ throughout, but found similar results for values in the range $10^{-7}-10^{-9} h^{5} \mathrm{Mpc}^{-5}$.

Since the SDSS has an isotropic selection function within the survey footprint, equation (22) is separable; we plot the radial and angular parts of the wave-function, $\psi(\mathbf{r})$ in Figure 2, where $\psi(\mathbf{r}) \equiv \psi(r) \psi(\theta)$. The actual footprint of the survey has a small but finite ellipticity, $e \simeq 0.1$. Rather than introducing a third, azimuthal, coordinate in solving Schrödinger's equation, we treat the angular shape of the survey as circular, and then simply stretch the wave-function appropriately.

\section{3. $\quad$ Isolating the Independent Modes}

We have made our choice of modes in order to minimize the off-diagonal elements in the covariance matrix. However, we have not guaranteed that all of our modes are linearly independent. If the survey volume is $V$, then we can imagine gridding the density distribution of the survey into $N^{3}$ cells. For large $N$, we can fully describe the density field on scales of $L / N$ with $f_{V} \times N^{3}$ numbers, where:

$$
f_{V} \equiv \frac{V}{L^{3}} .
$$

Thus we expect only a fraction, $f_{V}<1$, of our Fourier modes to be independent on small scales. For the geometry of the SDSS, this fraction is given by $f_{V}=0.176$.

Since, in general, this suggests that the covariance matrix is singular, we can use singular value decomposition (SVD), to create a new basis set. Examining the first $n$ 
Fourier modes, we determine two unitary matrices, $\mathbf{u}$ and $\mathbf{v}$ such that

$$
\mathbf{C}=\mathbf{u} \mathcal{C} \mathbf{v}^{\dagger}
$$

where $\mathcal{C}$ is a diagonal $n \times n$ matrix containing the eigenvalues, $s_{i}$, of $\mathbf{C}$ in decreasing order. Thus, by truncating at some critical value of $s_{m} / s_{1}$, we may look at only the first $f_{s}=m / n$ modes. For very large values of $n$, it is our expectation that $f_{s}$ approximately approaches $f_{V}$, since both represent a quantitative account of the amount of information per Fourier mode. In general, however, $f_{s}<f_{V}$ at large $n$, because ostensibly independent modes may be of such low signal to noise ratio that SVD rejects them.

We label $\mathcal{C}_{m}$ as the upper $m \times m$ elements of $\mathcal{C}$, as all other elements are very close to zero. We define the $n \times m$ matrices $\mathbf{u}_{m}$ and $\mathbf{v}_{m}$ as the first $m$ column vectors of $\mathbf{u}$ and $\mathbf{v}$, respectively. These matrices can be used to transform an $n \times n$ matrix into an $m \times m$ matrix. We can similarly transform any arbitrary matrix, $\mathbf{D}$ to the basis set by:

$$
\mathcal{D}_{m}=\mathbf{u}_{m}^{\dagger} \mathbf{D} \mathbf{v}_{m}
$$

By transforming $\partial \mathbf{C} / \partial \theta_{i}$ in this way, we can readily compute the matrices $\mathbf{A}_{i}=\mathcal{C}_{m}^{-1} \mathcal{C}_{m, i}$, where $\mathcal{C}_{m, i}$ is the transform of $\partial \mathbf{C} / \partial \theta_{i}$, and $\left(\mathcal{C}_{m}^{-1}\right)_{a b}=\delta_{a b}^{K} / s_{a}$, from which follows the Fisher matrix.

In order to determine the condition number $s_{m} / s_{1}$ at which $f_{s}$ approaches $f_{V}$, we look at the distribution function of eigenvalues of the covariance matrix. Figure 3 shows this distribution for covariance matrix sizes $n=63,342$, and 999, corresponding to $k=(3,6,9) \times 2 \pi / L$. This was done assuming $P(k)=$ constant and ignoring shot noise, because we want to probe the geometry of the survey, and not a particular power spectrum model. The horizontal dashed line is drawn at $f_{V}=0.176$, which crosses the $n=999$ line at $s_{m} / s_{1}=0.004$. For $n>342$, the distribution function asymptotes to a uniform curve, and therefore we can choose the condition number to be 0.004 independent of our value of $n$, and match the requirement $f_{s} \simeq f_{V}$.

\subsection{The Size of the Covariance Matrix}

Calculating the Fisher matrix requires performing an SVD on the covariance matrix. If we make no simplifying assumptions, a grid of $N$ different values of $\mathbf{k}$ requires an $N \times N$ matrix. Performing the SVD requires $\simeq N^{2}$ calculations, and transforming a matrix to the basis set requires $\simeq N^{3}$ calculations, not to mention the fact that each element in the matrix is a three dimensional integral over a non-symmetric function (equation 18)! For a survey of 
the size of the SDSS ( $\sim 10^{6}$ particles $)$, it is reasonable to have as many independent modes, and using the method outlined above, vastly overwhelm the limits of both computing time and of storage.

However, our task may be simplified somewhat. We have reason to suspect that that after some critical mode, $n_{\text {crit }}$, we may approximate the covariance matrix as being approximately diagonal, since at small scales, the convolution in equation (20) becomes more and more an unbiased estimator of $P(k)$, as the edge effects become less and less important.

Figure 7 represents the increasing sparseness of the covariance matrix pictorially, where we assume the SDSS geometry and selection function, again using the simplified model of a constant power spectrum with no shot noise. In Figure $4 a$, we show the first 10000 modes of the covariance matrix (selecting each $100^{\text {th }}$ mode). Figure $4 \mathrm{~b}$ shows the covariance matrix for the first 100 terms, and Figure $4 \mathrm{c}$ shows the $1000^{\text {th }}$ to $1100^{\text {th }}$ terms. In each case, the shading is linearly proportional to the covariance between the modes. The off-diagonal terms become smaller for the higher modes as the edge effects become less important. We should therefore be able to truncate analysis of the full covariance matrix at some point, as we discuss in the following subsection.

However, we cannot simply consider the covariance matrix to be completely diagonal beyond some $n_{\text {crit }}$, given the argument in $\S 3.3$ about the overcounting of independent modes. We therefore consider a likelihood function for the $n_{\text {diag }}$ modes from $n_{\text {crit }}+1$ to $n_{\text {crit }}+n_{\text {diag }}$, using the diagonal covariance matrix approximation:

$$
\mathcal{L}^{\text {diag }}=\frac{f_{V}}{2} \sum_{n=n_{\text {crit }}+1}^{n_{\text {crit }}+n_{\text {diag }}}\left(\frac{\left|d_{n}\right|^{2}}{C_{n n}}+\ln C_{n n}\right)+\frac{f_{V}}{2} n_{\text {diag }} \ln (2 \pi) .
$$

From this, the diagonal estimate Fisher matrix elements are:

$$
F_{i j}^{\text {diag }}=\frac{f_{V}}{2} \sum_{n=n_{\text {crit }}+1}^{n_{\text {crit }}+n_{\text {diag }}} \frac{\partial C_{n n} / \partial \theta_{i}}{C_{n n}} \frac{\partial C_{n n} / \partial \theta_{j}}{C_{n n}} .
$$

Thus, we will treat the covariance matrix in two parts: a square matrix $\mathbf{C}^{\text {square }}$ with $k<k_{n_{\text {crit }}}$, and a diagonal matrix $\mathbf{C}^{\text {diag }}$ with $k \geq k_{n_{\text {crit }}}$. Simple algebra demonstrates $\mathbf{F}=\mathbf{F}^{\text {square }}+\mathbf{F}^{\text {diag }}$. We now turn to the determination of an appropriate value for $n_{\text {crit }}$. 


\subsection{At What Point May We Use the Diagonal Covariance Matrix?}

In order to determine at what $n_{\text {crit }}$ we can start treating the covariance matrix as diagonal, we have computed both the full and diagonal covariance matrices for a power spectrum model with $\Omega_{0}=1, \Omega_{b}=0.057$, and $\sigma_{8}=1.6$, with the effects of shot noise included. We performed an SVD on the full covariance matrix using $s_{m} / s_{1}=0.004$ determined above. We then computed the Fisher matrix element corresponding to the normalization of the power spectrum for both the full and diagonal estimates, as a function of maximum mode number, $n$.

In order to compare these two estimates, let us define the Fisher function $F_{i i}(n)$ as the value of $F_{i i}$ computed for the first $n$ terms of the covariance matrix. To compare $F_{i i}$ computed using the full and diagonal covariance matrices, we introduce an improvement function:

$$
\mathcal{I}(n)=\frac{F_{i i}^{\text {diag }}(n)}{F_{i i}^{\text {full }}(n)} .
$$

It is our hope, of course, that for some value of $n$, the improvement function tends to unity, after which we can safely apply only diagonal terms without a significant loss of information.

In Figure 5, we present the results of this exercise. Figure 5a shows the improvement function as a function of mode number, while Figure a b shows the behavior of the Fisher function, $F(n)$, for the full matrix (solid) and the diagonal elements only (dashed). The two curves approach one another at $n=728$.

We thus adopt $n_{\text {crit }}=728$, and treat the covariance matrix as diagonal for larger $l$. In summary, we have several reasons to argue that we can do this:

1. The covariance matrix becomes increasingly sparse at higher values of $|k|$ (Figure $\llbracket$ );

2. The Fisher information matrix using the diagonal and the full covariance matrix gives similar results at $n=728$ (Figure 5);

3. By $n=728$, the distribution function of eigenvalues (Figure [3), and therefore the condition number required to make $f_{s} \simeq f_{V}$, asymptotes to a constant value.

\section{Results}




\section{1. $\quad$ Expected $\Omega_{b}$ Uncertainties for Various Cosmologies}

In this section, we compute the uncertainties of cosmological parameters used in a typical model power spectrum using the Fisher information matrix. We will present 3 models, each with $h=0.65, n_{\text {prim }}=1$, and $\Lambda=0$. Model 1 is a flat universe $\left(\Omega_{0}=1\right)$ with a baryonic component consistent with nucleosynthesis estimates $\left(\Omega_{b}=0.024 h^{-2}\right)$ and a value of $\sigma_{8}=1.6$. Model 2 is a flat universe with no baryonic component and $\sigma_{8}=1.6$. Finally, Model 3 is an open universe, $\Omega_{0}=0.3$, with $\Omega_{b}=0.024 h^{-2}$ and $\sigma_{8}=0.64$. All three models are consistent with COBE normalization for their respective cosmologies (Bunn \& White 1997 and references therein). The power spectra of Models 1 and 3 were shown in Figure 1.

We also include a Bayesian prior Fisher matrix, which takes into account the fact that we $d o$ have prior information about $h, n_{\text {prim }}$, and $\Omega_{0}$. We assume that these quantities have Gaussian-distributed, uncorrelated (and quite conservative) errors $\Delta \Omega_{0}=3, \Delta h=0.5$, and $\Delta n_{\text {prim }}=1$. The prior log-likelihood function is:

$$
\mathcal{L}^{\text {prior }}(\boldsymbol{\Theta})=\sum_{i}\left(\frac{\left(\theta_{i}-\theta_{i, 0}\right)^{2}}{\Delta \theta_{i, 0}^{2}}+\ln \left(\Delta \theta_{i, 0}\right)\right)+N \ln (2 \pi) .
$$

The contribution to the Fisher matrix is:

$$
F_{i j}^{\text {prior }}=\Delta \theta_{i, 0}^{-2} \delta_{i j}^{K}
$$

where $F_{i j}^{\text {total }}=F_{i j}^{S D S S}+F_{i j}^{\text {prior }}$.

Note that as we add data, the measured uncertainties will quickly dominate, and hence our final error estimates will not be strongly linked to our Bayesian priors.

Figure 6 shows $\Delta \theta_{i}=\left(F^{-1}\right)_{i i}^{1 / 2}$, the computed parameter uncertainties for each of these models. If we include all the Fourier modes down to a physical scale of $20 h^{-1} \mathrm{Mpc}$ (where the evolution of the power spectrum is expected to become non-linear), we find that the uncertainty in $\Omega_{b}$ is $0.070,0.060$, and 0.016 for the three models, respectively. Models 1 and 3 have a value of $\Omega_{b}=0.057$, which means that, at the very least, we could check for inconsistency with $\Omega_{b}=0$ at the 0.8 and $3.5 \sigma$ level, respectively. This suggests that studying redshift surveys in an open universe will give us a much better handle on $\Omega_{b}$ than will a closed universe, as the size of the wiggles depends on $\Omega_{b} / \Omega_{0}$ (Figure 1).

Note that $\Delta \sigma_{8}$ varies by a factor of $\simeq 2$ from the open to closed models, roughly the ratio of the values of $\sigma_{8}$ used in each model. This gives a fractional uncertainty which is roughly constant between models. Likewise, we estimate similar fractional uncertainties in $\Omega_{0}$ for all three models. 
Finally, it worth noting that there is no discontinuity in the behavior of any of the estimated parameter uncertainties at $n=728$ modes, where we start treating $\mathbf{C}$ as diagonal. This reassures us that the transition to a diagonal covariance matrix does not introduce any gross feature in the Fisher matrix.

\section{2. $\quad$ A Different Set of Parameters}

The parameters used in the parameter estimation, $\boldsymbol{\Theta}$, are not independent of each other; for example, $\Omega_{0}, h$, and $\Omega_{b}$ are coupled through $\Gamma$ (equation 2). This is reflected in the off-diagonal elements of the Fisher matrix, which is large for pairs that are coupled. Thus the covariance between $\Omega_{0}$ and $\Omega_{b}$,

$$
\mu_{i j} \equiv \frac{\left(F^{-1}\right)_{i j}}{\left[\left(F^{-1}\right)_{i i}\left(F^{-1}\right)_{j j}\right]^{1 / 2}},
$$

is $30 \%$ on small scales, and appreciably larger on larger scales. We thus are motivated to use a set of parameters that are closer to orthogonal, to minimize their uncertainty. In

particular, the parameter $\Gamma$ sets the overall shape of the power spectrum, and the amplitude of the wiggles goes roughly as $\Omega_{b} / \Omega_{0}$. Therefore, we now consider the parameter set, $\mathbf{T}=\left\{\sigma_{8}, \Gamma, \Omega_{b} / \Omega_{0}, h, n_{\text {prim }}\right\}$, since these quantities should be closer to independent.

We use the chain rule to convert between one data set and another:

$$
F_{a b}=\left\langle\frac{\partial^{2} \mathcal{L}}{\partial T_{a} \partial T_{b}}\right\rangle=\sum_{i, j} F_{i j} \frac{\partial \theta_{i}}{\partial T_{a}} \frac{\partial \theta_{j}}{\partial T_{b}} .
$$

Figure 7 plots the results of the parameter rotation. The uncertainties in $\Gamma$ are about three times larger in the flat universe models than the open universe model, corresponding to very similar fractional errors in each model. The uncertainties of the parameter $\Omega_{b} / \Omega_{0}$ behave consistently with the results determined in the previous section. That is, all three models produce roughly the same uncertainty in $\Omega_{b} / \Omega_{0}$, about 0.06 , corresponding to the values of $\Omega_{b} / \Omega_{0}$ inconsistent with zero at the $\simeq 1 \sigma$ level in Model 1 , and the $\simeq 3 \sigma$ level in Model 3. Note that models 1 and 2 have sharp downturns in $\Delta \Gamma$ at around the threshold of the nonlinear regime. This is due primarily to the fact that the wiggles appear most prominently in flat cosmologies at around those scales (see Figure (1). The covariance between $\Omega_{b} / \Omega_{0}$ and $\Gamma$ is only $15 \%$ in this regime, and thus both parameters can be determined with greater accuracy. 


\subsection{A Comparison with Band Estimates of the Power Spectrum}

We check for consistency of the above method with another, perhaps more intuitive estimate of the power spectrum. In this section, we divide $k$-space into bands, and determine the errors of $P(k)$ in each band. From there, we can compute the uncertainties in the power spectrum parameters using the Fisher formalism.

FKP give an estimate for the uncertainty in $\mathrm{P}(k)$ for an approximately isotropic survey. Though this is not strictly true in our case, this approach is merely to serve as an order of magnitude consistency check on the final errors. Moreover, on smaller scales, isotropy is a fairly good assumption. The expected error for each band is:

$$
\frac{\langle\Delta P(k)\rangle}{P(k)}=\left(\frac{(2 \pi)^{3} \int d^{3} \mathbf{r} \bar{n}^{4}(\mathbf{r}) \psi^{4}(\mathbf{r})\left[1+\frac{1}{\bar{n}(\mathbf{r}) P(k)}\right]^{2}}{V_{k}\left[\int d^{3} \mathbf{r} \bar{n}^{2}(\mathbf{r}) \psi^{2}(\mathbf{r})\right]^{2}}\right)^{1 / 2},
$$

where $V_{k}$ is the volume of the shell in $k$-space; we will use the weight function $\psi$ shown in Figure 2. If we take spherical shells, the volume of the $i^{\text {th }}$ shell is $V_{k}=\frac{4}{3} \pi\left(k_{i}^{3}-k_{i-1}^{3}\right)$. We set the bands to be spaced at increments of $k=2 \pi / L$, where $L$ is the characteristic length defined in $\S$ 3.1, as the modes should be approximately independent with this spacing. The FKP model ignores covariance between modes, and we will use this approximation here as well.

The Model 1 power spectrum $\left(\Omega_{0}=1, \Omega_{b}=0.057\right)$ and the resulting errors are plotted in Figure 8.

The log-likelihood function for some dataset of band-averaged power spectrum modes can be determined using a standard $\chi^{2}$ expression because we have neglected covariance:

$$
\mathcal{L}^{F K P}=\frac{1}{2} \sum_{l}\left(\frac{\left(P_{l}-\tilde{P}_{l}\right)^{2}}{\left(\Delta P_{l}\right)^{2}}+\ln \left(\Delta P_{l}\right)\right)+\frac{N}{2} \ln (2 \pi) .
$$

Here, $P_{l}$ is the value of $P\left(k_{l}\right)$ computed using a model, $\tilde{P}_{l}$ is the measured value of the power spectrum within the $l^{\text {th }}$ band, and $\Delta P_{l}$ is the associated error computed above. The Fisher information matrix follows straightforwardly (see also Tegmark 1997):

$$
F_{i j}=\sum_{l} \frac{\partial P_{l}}{\partial \theta_{i}} \frac{\partial P_{l}}{\partial \theta_{j}} \frac{1}{\left(\Delta P_{l}\right)^{2}}
$$

Note that $F_{i j}$ is insensitive to bin size; if we were to take smaller bins, we would have more terms contributing to the sum in equation (35), each with smaller $V_{k}$ and therefore 
larger $\Delta P$ (equation 33). In Figure 8, we present the comparison of the final errors on $\Omega_{b}$, $\sigma_{8}$, and $\Omega_{0}$ determined in this way with those determined using the full covariance matrix, as a function of the maximum value of $|k|$ used in the analysis. The two are in fairly good agreement, but in general, the band estimate produces smaller estimated uncertainties than does the covariance matrix analysis. This is not surprising, since the band estimates assume that each band can be measured independently from one another, and thus, it neglects covariance between different bands. This serves to underestimate the true error. Furthermore, the FKP approximation uses the assumption of isotropy and weighs all of the modes within a band equally (see Tegmark 1995). Finally, in the full covariance matrix analysis, covariance between individual modes within a given band can give information about $P(k)$. However, both the FKP approximation and the diagonal covariance matrix analysis ignore this.

\section{Other Complicating Factors}

We have assumed that the observed galaxy field reflects the mass density field on the length scales that we probe. Furthermore, we assumed that the redshifts measured represent the actual distances of the galaxies, and thus we have an accurate, three-dimensional map of the density field. We also assumed that the clustering of our density field behaves in the same way throughout the volume of the survey. Finally, we assumed that linear theory describes the evolution of the power spectrum perfectly down to some scale. We briefly address the validity of these assumptions, and what may be done to compensate for them when they do not hold. A more comprehensive review of some of these issues appears in Strauss \& Willick (1995).

\subsection{Evolution of Clustering}

The SDSS survey will extend to a redshift of $z \simeq 0.2$. As a result, we would expect that local structure will have a clustering amplitude which is larger than at the furthest reaches of the survey due to the growth of structure with time. Let $A(z)$ be the ratio of power at redshift, $z$, to redshift $0 ; A(z)=1 /(1+z)^{2}$ for $\Omega_{0}=1$, in linear theory. We can correct the density perturbations at any given redshift, $z$, to account for the cluster evolution that will take place between $z$ and $z=0$ :

$$
\hat{\delta}(\mathbf{k})=\int d^{3} \mathbf{r} e^{i \mathbf{k} \cdot \mathbf{r}} A(z)^{-1 / 2} \psi(\mathbf{r}) \delta(\mathbf{r}),
$$

and where $z$ and $r$ are related through the standard cosmological equations. 
Following this through, we find that the window function is redefined as:

$$
W(\mathbf{k})=\int d^{3} \mathbf{r} e^{i \mathbf{k} \cdot \mathbf{r}} A(z)^{-1 / 2} \psi(\mathbf{r})
$$

Using the revised definition of the window function, the covariance matrix becomes

$$
C_{l m}=\left\langle\hat{\delta}_{l} \hat{\delta}_{m}\right\rangle=\frac{1}{(2 \pi)^{3}} \int d^{3} \mathbf{k}^{\prime} P\left(\mathbf{k}^{\prime}\right) W\left(\mathbf{k}_{l}-\mathbf{k}^{\prime}\right) W\left(\mathbf{k}_{m}-\mathbf{k}^{\prime}\right)^{*}+\int d^{3} \mathbf{r} A(z)^{-1} \frac{\psi(\mathbf{r})^{2} e^{i\left(\mathbf{k}_{l}-\mathbf{k}_{m}\right) \cdot \mathbf{r}}}{\bar{n}(\mathbf{r})} .
$$

$A(z)$ depends on $\Omega_{0}$ and to a lesser degree $\Lambda$. Therefore, including this effect breaks the degeneracy between $\Omega_{0}$ and $h$, although further experiments need to be done to quantify how large an effect this actually is.

\subsection{Redshift Space Distortions}

Throughout this paper, we have assumed that we know the full three-dimensional position of each galaxy. However, in a real survey, we do not know the distance of each galaxy, only its redshift, which differ due to peculiar velocities. On small scales, the redshift distribution differs from the real space distribution via the "fingers of God", the elongated structures seen along the line of sight due to motions of galaxies in a virialized system. On larger scales, gravitational collapse causes structure to appear compressed along the line of sight.

If our galaxy survey were confined to a sufficiently small angle on the sky, we would be able to use a fairly simple approximation in linear theory to account for these effects (Kaiser 1987; Cole, Fisher, \& Weinberg 1994):

$$
\hat{\delta}(\mathbf{k})_{S}=\hat{\delta}(\mathbf{k})_{R}\left(1+\beta \mu^{2}\right),
$$

where $\hat{\delta}_{S}$ and $\hat{\delta}_{R}$ are the Fourier transforms of the redshift and real density perturbations, respectively, $\beta \equiv f\left(\Omega_{0}\right) / b$, where $b$ is the biasing parameter, and $f\left(\Omega_{0}\right)$ is the logarithmic derivative of the fluctuation growth rate, and the cosine of the angle between $\mathbf{k}$ and the line of sight is $\mu$.

The more general case is discussed by Zaroubi \& Hoffman (1996). Using linear perturbation theory, they find the general relation between the redshifted and real Fourier modes. They show that different values of $\mathbf{k}$ become coupled due to redshift distortions. Since we actually "measure" $\hat{\delta}(\mathbf{k})_{S}$, but the real density perturbations are given by $\hat{\delta}(\mathbf{k})_{R}$, one needs to compute the covariance matrix of the redshift space modes in terms of the 
real space perturbations and their redshift distortions. This of course introduces a new parameter, $\beta$, to be included in the Fisher matrix. The expressions of Zaroubi \& Hoffman (1996) are quite complicated, and introduce further coupling between modes, which may affect the applicability of the techniques used in this paper. In the Appendix, we discuss an alternate basis set that has the potential to reduce this problem for redshift space distortions.

Finally, Nakamura et al. (1997) and de Laix \& Starkman (1997) discuss the effects of space curvature on the redshift space distortions in the SDSS galaxy survey, and show that these effects need to be taken into account for a proper treatment of redshift space distortions.

\section{3. $\quad$ Biasing and Selection Effects}

We have further ignored the fact that we are dealing with the galaxy density field, rather than the unbiased matter density field. Here we mention several effects that biasing may give.

First, we have assumed that a galaxy density fluctuation of a certain scale, $\delta_{g}(\mathbf{r})$ is equal to the density fluctuation of matter, $\delta_{D M}(\mathbf{r})$. Indeed, the two may be related by some constant of proportionality, $b$, such that $\delta_{g a l}(\mathbf{r})=b \delta_{D M}(\mathbf{r})$. The normalization parameter, $\sigma_{8}$, which is proportional to $P(k)^{1 / 2}$, is also proportional to $b$, and thus is not a major concern.

A more serious issue comes from the fact that biasing is not necessarily linear, nor is it deterministic. The bias can be a strong function of scale and can have finite scatter (cf., Cen \& Ostriker 1992), and hence, the normalization (and the other relevant parameters) are no longer corrected in a non-trivial way.

We have further made the assumption that the bias is independent of galaxy luminosity. Since the galaxies at high redshift are at the bright end of the luminosity distribution in a flux-limited sample, the measured galaxy power spectrum at high $z$ will be different from that found locally if this assumption breaks down. We could avoid this problem by using a volume-limited survey. However, this would increase the shot noise; further calculations are needed of the Fisher matrix in this case to see how this affects $\Delta \Omega_{b}$.

Finally, we have made the assumption that there is no substantial evolution in galaxy populations since $z \simeq 0.2$. This further complicates matters in that the bias parameter can be a function of $z$ (e.g., Fry 1996) as well as local density. 
These objections notwithstanding, future improvement in our understanding of evolution, biasing, and the universal luminosity function can certainly be incorporated into the Fisher formalism. Once these effects are included in the covariance matrix, the error estimation proceeds exactly as we have seen, with the possible addition of further parameters which describe these effects.

\subsection{Nonlinear Effects}

We have treated the evolution of the power spectrum as well described by linear theory down to a scale of $20 h^{-1} \mathrm{Mpc}$, and assumed that everything below that scale was nonlinear. However, this may not be a concrete limit. Both analytic (Taylor \& Hamilton 1996; Jain \& Bertschinger 1994) and numerical (Baugh \& Efstathiou 1994) analyses suggest that for some models, the linear regime may extend to even smaller scales. This produces two competing effects. First, nonlinearity tends to smooth out features in the power spectrum, reducing the size of the wiggles, and thus, limiting the information about $\Omega_{b}$. However, if we have a good model of the power spectrum which does extend well into the non-linear regime, we can also extend our covariance matrix to smaller scales. Though we expect features like the wiggles to be smoothed out (cf., Tegmark 1997), we nevertheless may expect to get more information on $\Gamma$, and with that parameter more tightly constrained, our uncertainties in $\Omega_{b}$ decrease as well due to the covariance between them.

\section{Conclusions}

We have discussed the possibility of measuring $\Omega_{b}$ from the power spectrum in the forthcoming SDSS. In order to simplify matters, we have assumed uniform biasing, and that there are no redshift space distortions and no evolution of either the density fields or of the constituent galaxies. Our approach is to use the Fisher information matrix, which uses the covariance matrix of the data to put minimum error bars on derived parameters via the Cramèr-Rao inequality.

We decompose the density field of a galaxy redshift survey into discrete Fourier modes in units of $2 \pi / L$, where the characteristic length, $L=1466 h^{-1} \mathrm{Mpc}$, is given by the survey geometry. The data are placed in order of decreasing physical scale. This ordering is ideal in the sense that a given mode number is associated with a physical scale, and the signature of a high baryon density (the wiggles) are scale dependent.

Computational limits do not allow us to consider the full covariance matrix for more 
than $\sim 1000$ Fourier modes, corresponding to $k=9 \times 2 \pi / L$. We show, however, that on these scales, the covariance matrix becomes accurately diagonal, and we use the diagonal approximation for $n>728$ modes. We take care to account for the correct number of independent modes, using singular value decomposition for $n<728$, and a calculation of the effective volume of our sample for larger $n$. Calculations are continued to physical scales of $20 h^{-1} \mathrm{Mpc}$, where it is expected that the evolution of the power spectrum becomes significantly nonlinear.

We have applied this method to several different models, including a flat $\left(\Omega_{0}=1\right)$, and an open $\left(\Omega_{0}=0.3\right)$ model, each with $\Omega_{b}=0.024 \pm 0.002 h^{-2}$, and a flat model with no baryons. In the models with baryons, we found that the uncertainties were inconsistent with $\Omega_{b}=0$ at the $0.8 \sigma$ level in a flat cosmology, and at the $3.5 \sigma$ level in an open cosmology. The quantities $\Omega_{b} / \Omega_{0}$, which describes the amplitude of the wiggles, and $\Gamma$, which describes the overall shape of the power spectrum, should be close to orthogonal in the fits. We found that $\Omega_{b} / \Omega_{0}$ is inconsistent with $\Omega_{b}=0$ at the $1 \sigma$ and $3 \sigma$ in the flat and open models, respectively. Finally, we compared this result with the estimate of uncertainties which can be derived from band estimates of the power spectrum and found qualitatively consistent results, all of which lends great confidence to the idea that in an open universe, $\Omega_{b}$ may be independently measured from galaxy redshift surveys.

We have ignored in this paper a number of physical effects that need to be understood before we can claim a definitive calculation of the power spectrum and its errors. We plan to use numerical simulations to include the effects of nonlinearities; if these can be properly modeled, it is possible that errors on parameters can be tightened considerably. Distortions to the density field due to galaxy peculiar velocities (redshift-space distortions) are known to be an important effect; including them would complicate the analysis considerably, although the basis set described in the Appendix may simplify the problem. The effects of space curvature and the evolution of clustering are probably less important, but are straightforward to include. Finally, scale- and luminosity-dependent galaxy biasing are additional complications which are difficult to model realistically, but it should be straightforward to give rough estimates for how important these effects are on the estimation of quantities such as $\Omega_{b}$.

The authors would like to gratefully acknowledge David Spergel, Michael Vogeley, Max Tegmark and Michael Blanton for invaluable discussions. We thank David Weinberg for the use of his mock catalog. M.A.S. acknowledges support from the Alfred P. Sloan Foundation. D.M.G. is supported by an NSF Graduate Research Fellowship. 


\section{A. An Alternate Basis Set}

Throughout the paper, we have used the Fourier modes of the density field as our basis set. However, we could imagine other basis sets that take the SDSS geometry better into account. D. Spergel (private communication) suggests using a form of spherical harmonics and spherical Bessel functions for the basis set, with basis vectors given by:

$$
\hat{e}_{l m n}=\mathcal{Y}_{l m}(\theta, \phi) j_{l}\left(k_{n} r\right)
$$

where

$$
\mathcal{Y}_{l m}(\theta, \phi)=\left[\frac{2 l+1}{4 \pi} \frac{(l-m) !}{(l+m) !}\right]^{1 / 2} P_{l}^{m}\left(\frac{\cos \theta}{\cos \theta_{0}}\right) e^{i m \phi} \sqrt{\cos \theta_{0}} .
$$

Here, $P_{l}^{m}$ are the Legendre polynomials, $\mathcal{Y}_{l m}$ are a set of functions related to the spherical harmonics, $j_{l}(x)$ are the spherical Bessel functions, $k_{n}$ is the $n^{\text {th }}$ value of $k$ satisfying $j_{l}\left(k_{n} R\right)=0$ at the edge of the survey, and $\theta_{0}$ is the radial angle of the circular cone which defines our space.

It is straightforward to show that this forms a complete, orthonormal basis set within a circular cone of opening half-angle $\theta_{0}$, embedded in a sphere of radius $R$. The SDSS geometry is fairly similar. Therefore, we might imagine that using such a basis set will give us a very nearly diagonal covariance matrix. The resolution scale of these modes are related to $n_{\max }$ and $l_{\max }$, the maximum values of $n$ and $l$, respectively (see Fisher et al. 1995 for a discussion).

We can first decompose the density field into modes of this basis set:

$$
\delta(\mathbf{r})=\sum_{l=0}^{l_{\max }} \sum_{m=-l}^{l} \sum_{n=1}^{n_{\max }} B_{l n} \hat{\delta}_{l m n} j_{l}\left(k_{n} r\right) \mathcal{Y}_{l m}(\theta, \phi),
$$

where $B_{l n}$ is a normalization constant. The transform conjugate is given by

$$
\hat{\delta}_{l m n}=\int d^{3} \mathbf{r} j_{l}\left(k_{n} r\right) \mathcal{Y}_{l m}^{*}(\theta, \phi) \delta(\mathbf{r}) .
$$

Without loss of generality, we can add a separable weighting function $\psi(\mathbf{r})=\psi(r) \psi(\theta, \phi)$. This is included analogously to the weighting function used with the Fourier modes, and indeed, the method suggested by Tegmark (1995) is separable in radial and angular parts, and thus may provide the best solution to limit covariance.

Following Fisher et al. (1993), the expectation value of the product of any two modes is:

$$
\left\langle\hat{\delta}_{l m n} \hat{\delta}_{l^{\prime} m^{\prime} n^{\prime}}^{*}\right\rangle=\int d^{3} \mathbf{r}_{1} \int d^{3} \mathbf{r}_{2} \xi\left(\mathbf{r}_{1}-\mathbf{r}_{2}\right) j_{l}\left(k_{n} r_{1}\right) j_{l^{\prime}}\left(k_{n^{\prime}} r_{2}\right) \mathcal{Y}_{l m}^{*}\left(\theta_{1}, \phi_{1}\right) \mathcal{Y}_{l^{\prime} m^{\prime}}\left(\theta_{2}, \phi_{2}\right)
$$




$$
\begin{aligned}
& \times \quad \psi\left(r_{1}\right) \psi\left(r_{2}\right) \psi\left(\theta_{1}, \phi_{1}\right) \psi\left(\theta_{2}, \phi_{2}\right) \\
& +\quad \int d^{3} \mathbf{r} \frac{j_{l}\left(k_{n} r\right) j_{l^{\prime}}\left(k_{n^{\prime}} r\right) \mathcal{Y}_{l m}^{*}(\theta, \phi) \mathcal{Y}_{l^{\prime} m^{\prime}}(\theta, \phi) \psi(r)^{2} \psi(\theta, \phi)^{2}}{\bar{n}(\mathbf{r})}
\end{aligned}
$$

We will label the latter term $C_{l m n l^{\prime} m^{\prime} n^{\prime}}^{s h}$.

Following Fisher et al. (1995), we use the Rayleigh expansion to find:

$$
e^{i \mathbf{k} \cdot \mathbf{r}}=\frac{4 \pi}{\cos \theta_{0}} \sum_{l, m} i^{l} j_{l}\left(k r \cos \theta_{0}\right) \mathcal{Y}_{l m}^{*}(\theta, \phi) \mathcal{Y}_{l m}\left(\theta_{k}, \phi_{k}\right)
$$

where $\theta_{k}$ and $\phi_{k}$ are the spherical coordinates of $\hat{\mathbf{k}}$.

Using this, and the definition of the autocorrelation function, we see:

$$
\begin{array}{r}
\xi\left(\mathbf{r}_{1}-\mathbf{r}_{2}\right)=\frac{2}{\pi \cos \theta_{0}} \sum_{l^{\prime \prime} m^{\prime \prime}} \mathcal{Y}_{l^{\prime \prime} m^{\prime \prime}}\left(\theta_{1}, \phi_{1}\right) \psi\left(\theta_{1}, \phi_{1}\right) \mathcal{Y}_{l^{\prime \prime} m^{\prime \prime}}\left(\theta_{2}, \phi_{2}\right) \psi\left(\theta_{2}, \phi_{2}\right) \\
\int k^{2} d k P(k) j_{l^{\prime \prime}}\left(k r_{1} \cos \theta_{0}\right) \psi\left(r_{1}\right) j_{l^{\prime \prime}}\left(k r_{2} \cos \theta_{0}\right) \psi\left(r_{2}\right)
\end{array}
$$

Plugging this in, we get:

$$
\begin{aligned}
C_{l m n l^{\prime} m^{\prime} n^{\prime}}= & \frac{2}{\pi \cos \theta_{0}} \sum_{l^{\prime \prime} m^{\prime \prime}} A_{l m l^{\prime \prime} m^{\prime \prime}} A_{l^{\prime} m^{\prime} l^{\prime \prime} m^{\prime \prime}}^{*} \int d k k^{2} P(k) \\
& \int d r_{1} r_{1}^{2} j_{l}\left(k_{n} r_{1}\right) j_{l^{\prime \prime}}\left(k r_{1} \cos \theta_{0}\right) \psi\left(r_{1}\right) \\
& \int d r_{2} r_{2}^{2} j_{l^{\prime}}\left(k_{n^{\prime}} r_{2}\right) j_{l^{\prime \prime}}\left(k r_{2} \cos \theta_{0}\right) \psi\left(r_{2}\right)+C_{l m n l^{\prime} m^{\prime} n^{\prime}}^{s h o t},
\end{aligned}
$$

where we have defined:

$$
A_{l m l^{\prime} m^{\prime}} \equiv \int d \omega \mathcal{Y}_{l m}^{*}(\theta, \phi) \mathcal{Y}_{l^{\prime} m^{\prime}}(\theta, \phi) \psi(\theta, \phi)
$$

In the case where we have perfect sky coverage over a circle of radius $\theta_{0}$ and uniform $\psi(\theta, \phi)$, the $A$ coefficients go to $A_{l m l^{\prime} m^{\prime}}=\delta_{l l^{\prime}}^{K} \delta_{m m^{\prime}}^{K}$, which simplifies the form substantially. Indeed, since there is only covariance between different values of $n$, the covariance matrix becomes block diagonal. Each block can be constructed as a covariance matrix of constant $l$ and $m$ and varying $n$. This would simplify storage and inversion tremendously, allowing us to use all the observed data.

Perhaps even more importantly, redshift distortions also only couple different values of $n$ (Fisher et al. 1994, 1995; Heavens \& Taylor 1995), and so this formalism should allow these distortions to be modeled without a great deal of additional complications. 
There are difficulties, however. First, the survey is not perfectly circular, and hence there is some covariance between different values of $l$ and $m$. Moreover, there is a double sum and a triple integral in this form of the covariance matrix (compared with only a triple integral in the Fourier picture). Moreover, the $A$ coefficients, though not model specific, still need to be computed for a given geometry, and stored in a four dimensional array. Finally, computing spherical Bessel functions and spherical harmonics are time consuming.

Still, this basis set offers the possibility of an even better approximation of diagonalizability than the Fourier modes, and may be useful for future consideration.

\section{REFERENCES}

Baugh, C. M., \& Efstathiou, G. 1994, MNRAS, 270, 183

Bunn, E. F., \& White, M. 1997, ApJ, 480, 6

Cen, R. Y., \& Ostriker, J. P. 1992, ApJ, 399, L113

Cole, S., Fisher, K. B., \& Weinberg, D. H. 1994, MNRAS, 267, 785

de Laix, A.A., \& Starkman, G. 1997, preprint (astro-ph/9707008)

Feldman, H. A., Kaiser, N., \& Peacock, J. A. 1994, ApJ, 426, 23 (FKP)

Fisher, K. B., Davis, M., Strauss, M. A., Yahil, A., \& Huchra, J. P. 1993, ApJ, 402, 42

Fisher, K. B., Lahav, O., Hoffman, Y., Lynden-Bell, D., \& Zaroubi, S. 1995, MNRAS, 272, 885

Fisher, K. B., Scharf, C. A., \& Lahav, O. 1994, MNRAS, 266, 219

Fry, J.N. 1996, ApJ, 461, L65

Gunn, J. E., \& Weinberg, D. H. 1995, in "Wide-Field Spectroscopy and the Distant Universe", ed. S. J. Maddox \& A. Aragón-Salamanca (Singapore: World Scientific), 3

Hamilton, A. J. S. 1997a, MNRAS, in press, astro-ph/9701008

Hamilton, A. J. S. 1997b, MNRAS, in press, astro-ph/9701009

Heavens, A.F., \& Taylor, A.N. 1995, MNRAS, 275, 483

Holtzman, J. A. 1989, ApJS, 71, 1

Hu, W., \& Sugiyama, N. 1996, ApJ, 471, 542

Jain, B., \& Bertschinger, E. 1994, ApJ, 431, 495

Kaiser, N. 1987, MNRAS, 227, 1 
Kundić, T., Turner, E. L., Colley, W. N., Gott, J. R., Rhoads, J. E., Wang, Y., Bergeron, L. E., Gloria, K. A., Long, D. C., Malhotra, S., \& Wambsganss, J. 1997, ApJ, 482, 68

Nakamura, T.T., Matsubara, T., \& Suto, Y. 1997, preprint (astro-ph/9706034)

Padmanabhan, T. 1995, "Structure Formation in the Universe" (Cambridge: Cambridge University Press)

Park, C., Vogeley, M. S., Geller, M. J., \& Huchra, J. P. 1994, ApJ, 431, 569

Peacock, J. A., \& Dodds, S. J. 1994, MNRAS, 267, 1020

Peebles, P. J. E. 1993, "Principles of Physical Cosmology" (Princeton: Princeton University Press)

Seljak, U., \& Zaldarraiga, M. 1996, ApJ, 469, 437

Strauss, M. A. 1997, in "Formation of Structure in the Universe", eds. A. Dekel and J. P. Ostriker (Cambridge: Cambridge University Press), in press, astro-ph/9610032

Strauss, M. A. \& Willick, J. A. 1995, Phys. Rep., 261, 271

Sugiyama, N. 1995, ApJS, 100, 296

Taylor, A. N., \& Hamilton, A. J. S. 1996, MNRAS, 282, 767

Tegmark, M. 1995, ApJ, 455, 429

Tegmark, M. 1997, preprint, astro-ph/97061982

Tegmark, M., Taylor, A., \& Heavens, A. F. 1997a, ApJ, 480, 22 (TTH)

Tegmark, M., Hamilton, A. J. S., Strauss, M. A., \& Vogeley, M. S. 1997b, to be submitted to ApJ

Tytler, D., Fan, X., \& Burles, S. 1996, Nature, 381, 207

Vogeley, M. S., \& Szalay, A. S. 1996, ApJ, 465, 34

White, M., Viana, P. T. B., Liddle, A. R., \& Scott D. 1996, MNRAS, 283, 107

Zaroubi, S., \& Hoffman, H. 1996, ApJ, 462, 25 

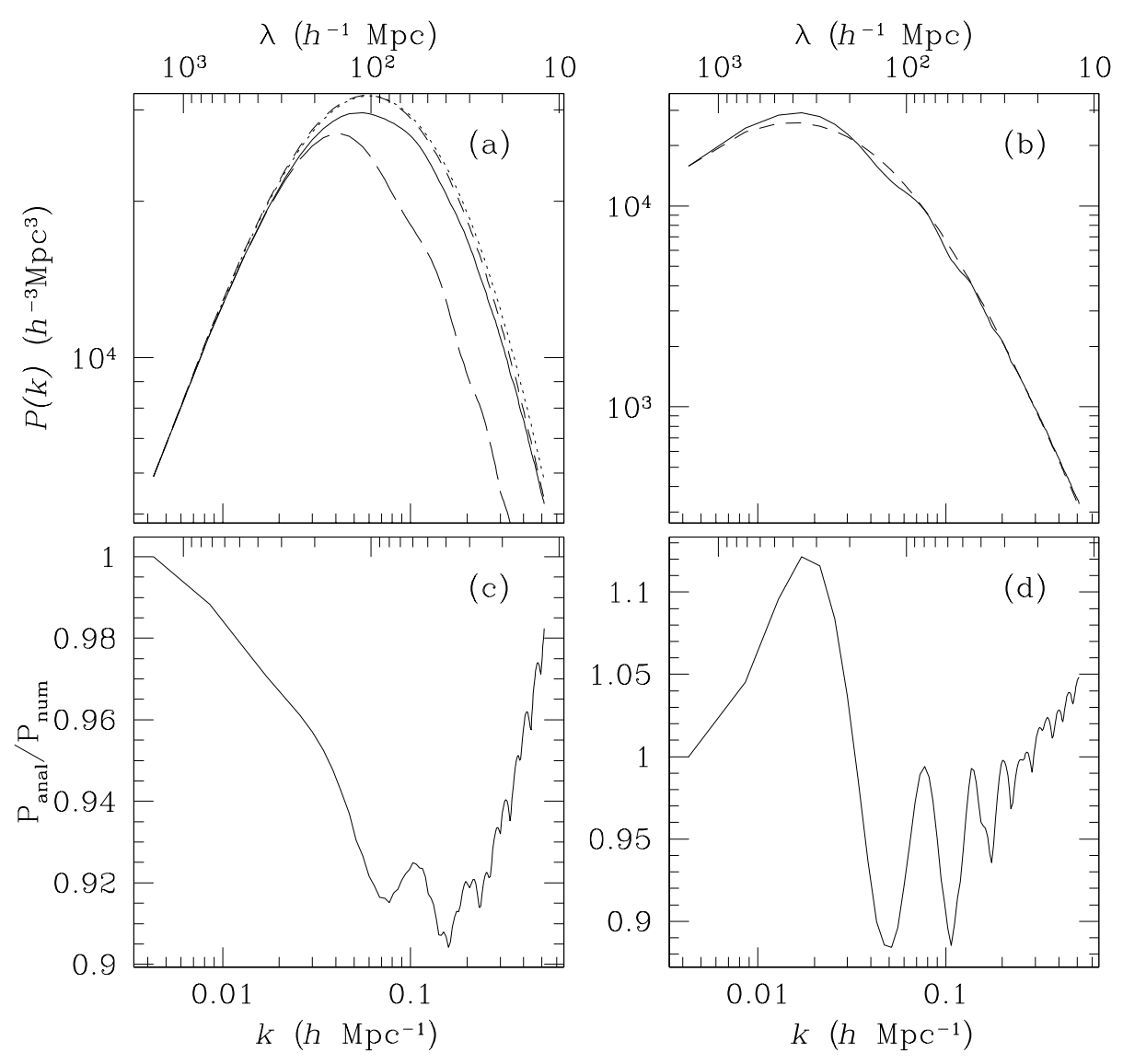

Fig. 1. - (a) Comparison of 4 power spectra, each with $h=0.65, n_{\text {prim }}=1$, and $\Gamma=0.58$. The solid line is the numerical result from CMBfast with $\Omega_{0}=1.0, \Omega_{b}=0.057$. The shortdashed line is the analytic power spectrum from equation 1 using the same parameters. The dotted line is the numerical result for $\Omega_{0}=0.89, \Omega_{b}=0$. The long-dashed line is the numerical result for $\Omega_{0}=1.37, \Omega_{b}=0.26$, corresponding to the same ratio of $\Omega_{b} / \Omega_{0}$ as in the open model in (b). (b) A comparison of the numerical (solid) and analytical (dashed) model power spectra for an open cosmological model, with $\Omega_{0}=0.3, \Omega_{b}=0.057, \Gamma=0.173$. (c) The ratio of the analytic to numerical power spectra for $\Omega_{0}=1$ and $\Omega_{b}=0.057$. (d) The ratio of the analytic to numerical power spectra for $\Omega_{0}=0.3$ and $\Omega_{b}=0.057$. 

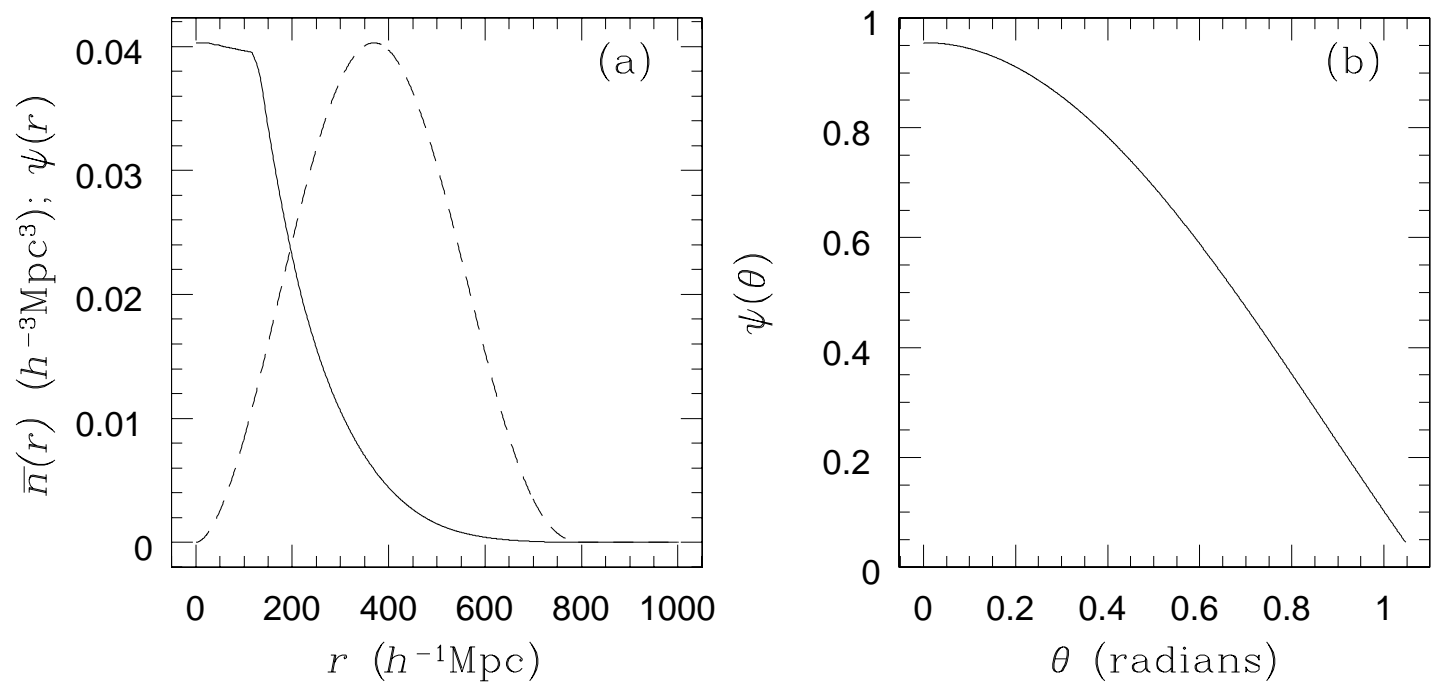

Fig. 2.- (a) The selection function (solid), and the radial weight $\psi(r)$ used in weighting the galaxy catalog. (b) The angular behavior of the weight, $\psi(\theta)$, where $\theta$ is the angle from the central axis of the survey cone. Since the radial and angular parts of the weighting function are completely separable, the normalization of each component is arbitrary. 


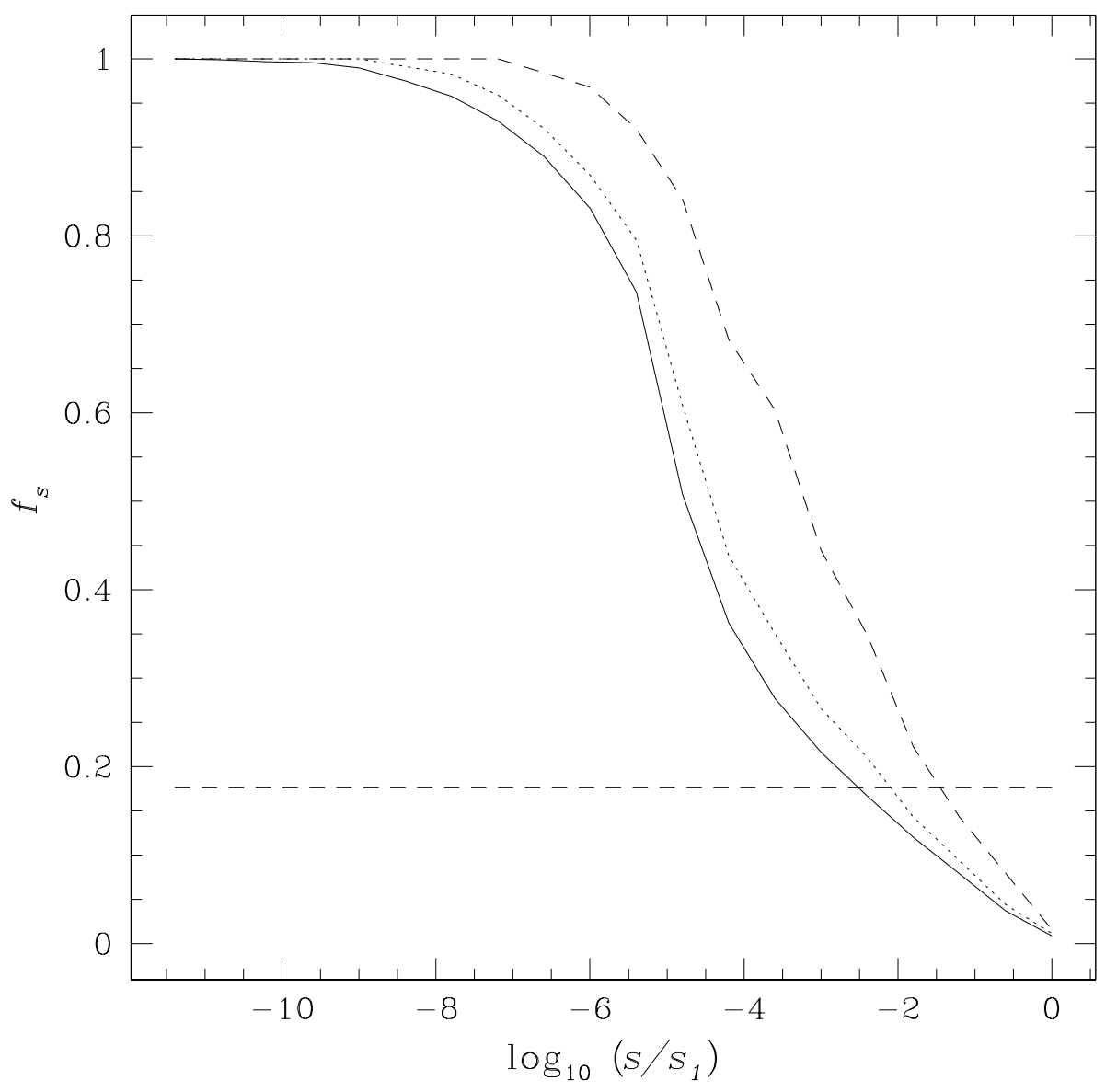

Fig. 3.- A plot of the fraction of eigenvalues $s$ that are greater than some fractional value, for several different maximum numbers of modes. The dotted line is 63 modes, the dashed line is 342 modes, and the solid line is 999 modes. The horizontal line represents $f_{V}=0.176$. As $n$ increases, the curves asymptote to a uniform form, and that at $n=999$ modes, a value of $s_{m} / s_{1}=0.004$ gives approximately $f_{s}=f_{V}$. 


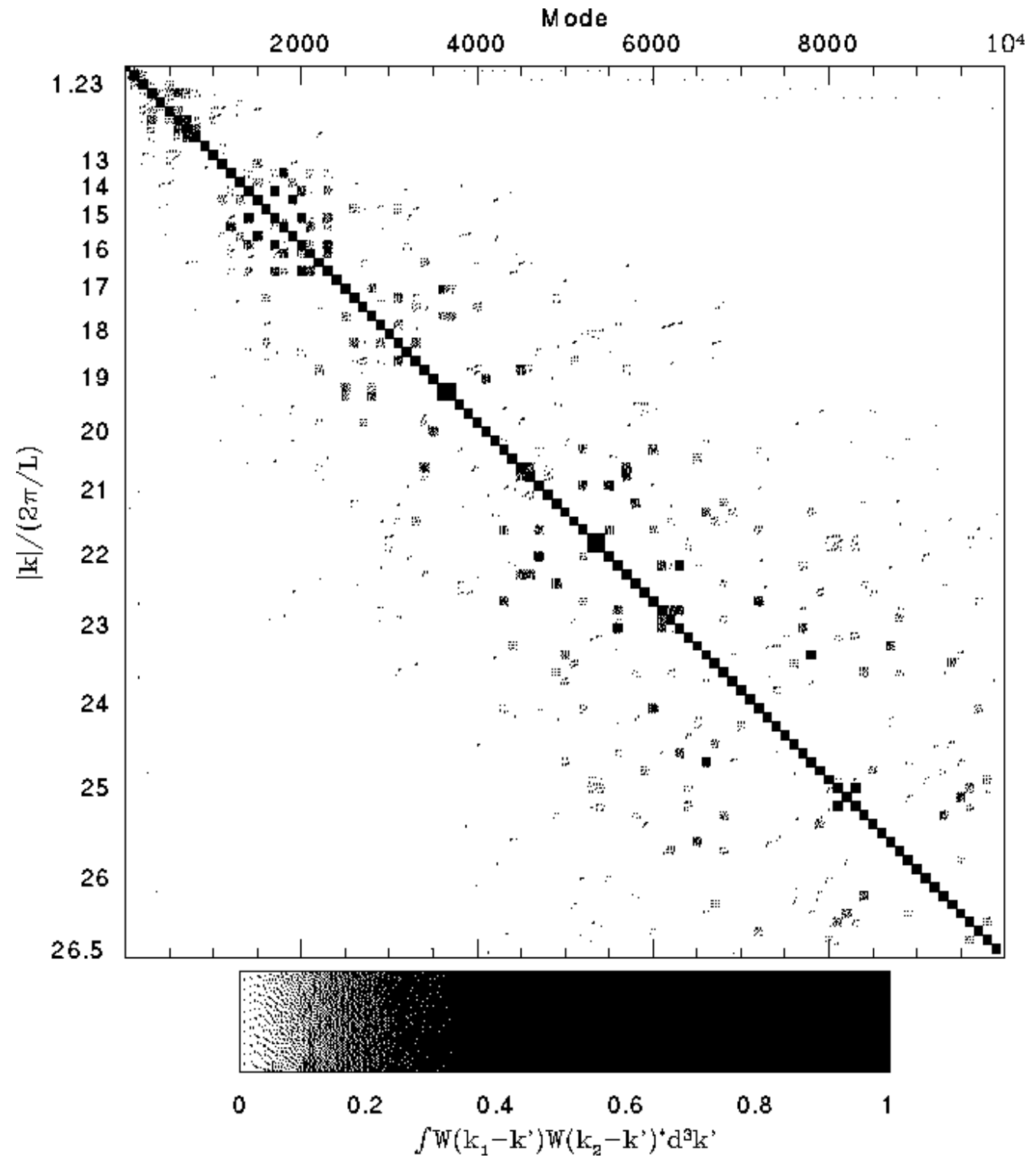




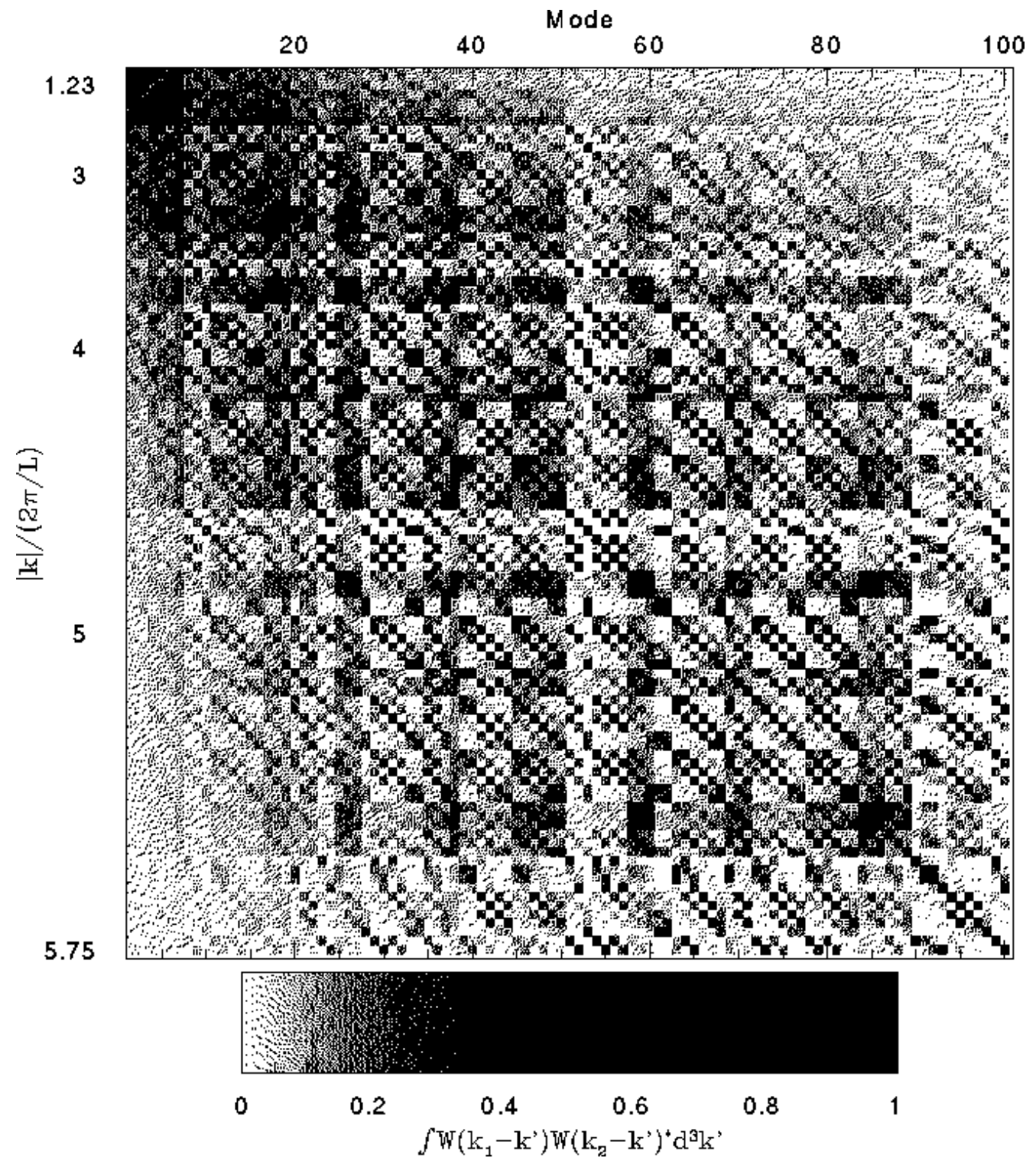




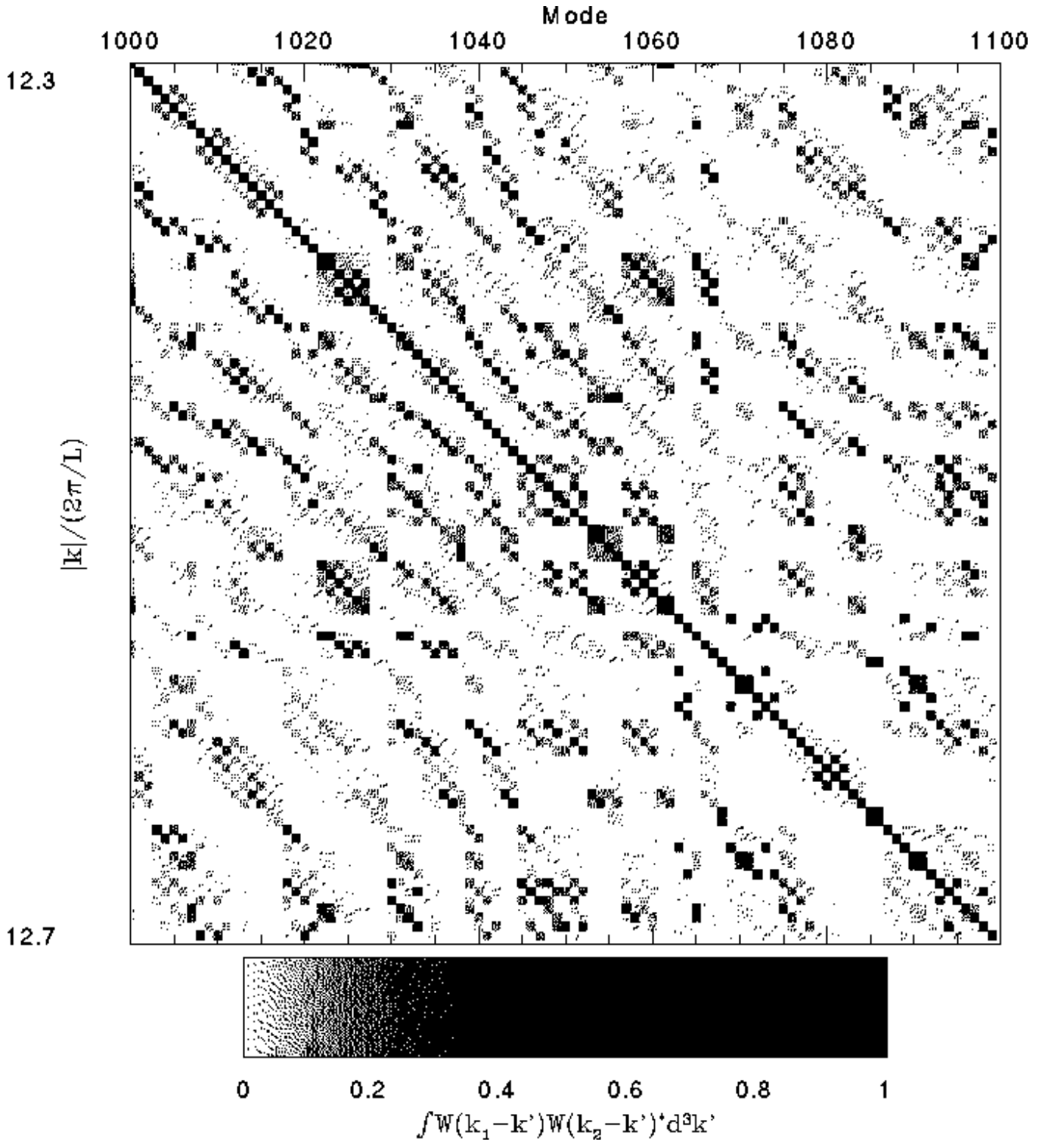

Fig. 4.- A grey-scale map of the weighting used in the covariance matrices, ordered in increasing value of $|k|$. The weighting goes as: $\int d^{3} \mathbf{k}^{\prime} W\left(\mathbf{k}^{\prime}-\mathbf{k}_{1}\right) W\left(\mathbf{k}^{\prime}-\mathbf{k}_{2}\right)^{*}$. (a) $1 / 100$ of the first $10^{4}$ modes. (b) The first 100 modes. (c)The $1000-1100^{\text {th }}$ modes. Note that the matrix gets sparser and sparser as we look at higher and higher modes. 

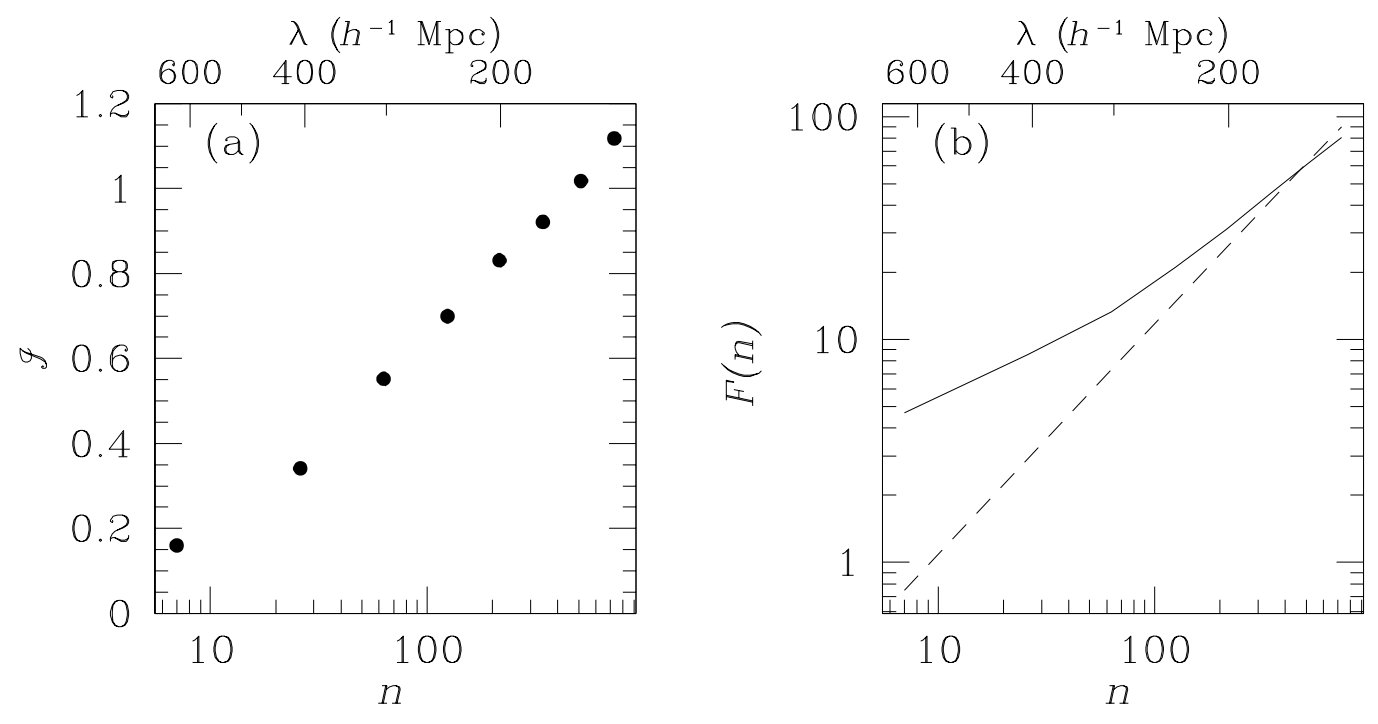

Fig. 5.- (a) The improvement function, $\mathcal{I}(n)$, for the normalization of the power spectrum as a function of the number of modes, $n$. (b) The Fisher function, $F(n)$. The solid line is computed using the full covariance matrix, while the dashed line is computed using the diagonal elements only. The above analysis goes out to $k=8 \times 2 \pi / L$, which corresponds to the first 728 modes. 

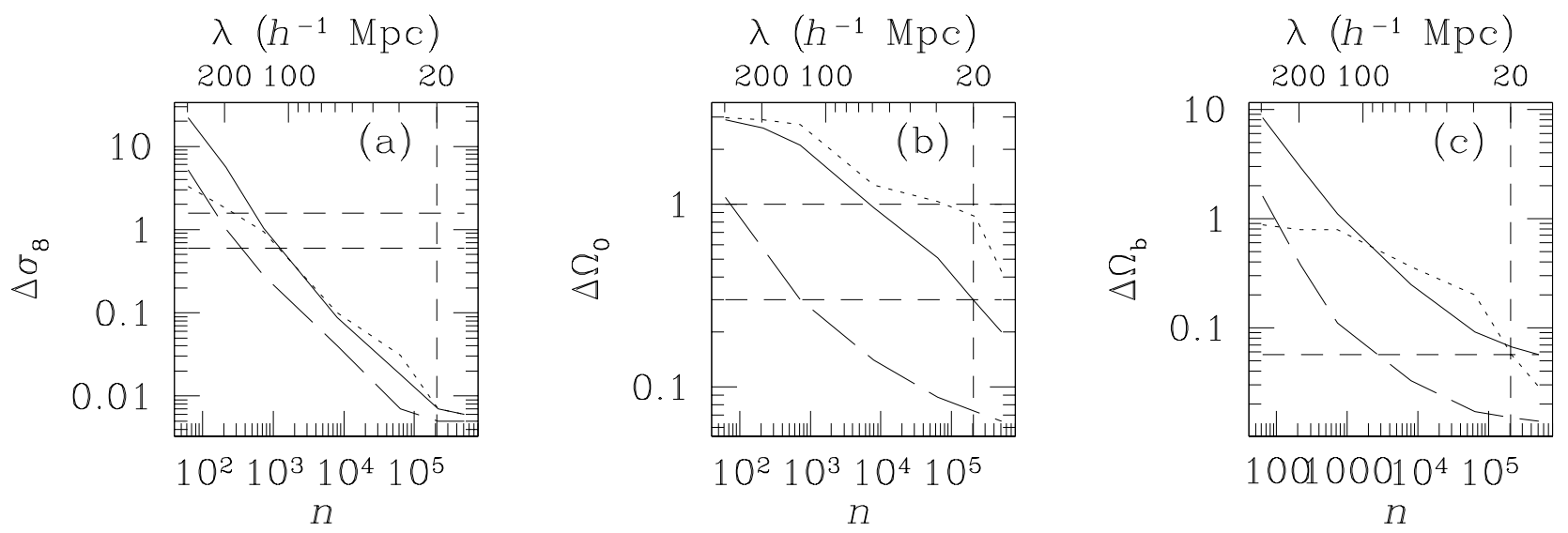

Fig. 6. - The computed uncertainties for (a) $\sigma_{8}$, (b) $\Omega_{0}$, and (c) $\Omega_{b}$ for various cosmological models. The solid line is Model 1, the flat, $\Omega_{b}=0.057$ model. The dotted line is Model 2, the flat $\Omega_{b}=0$ model. The long dashed line is Model 3 , the $\Omega_{0}=0.3, \Omega_{b}=0.057$ model. The short-dashed vertical line is at the mode corresponding to $\lambda=20 h^{-1} \mathrm{Mpc}$, the approximate limit of the linear regimes. The horizontal dashed lines are the actual values used in each of the models. 

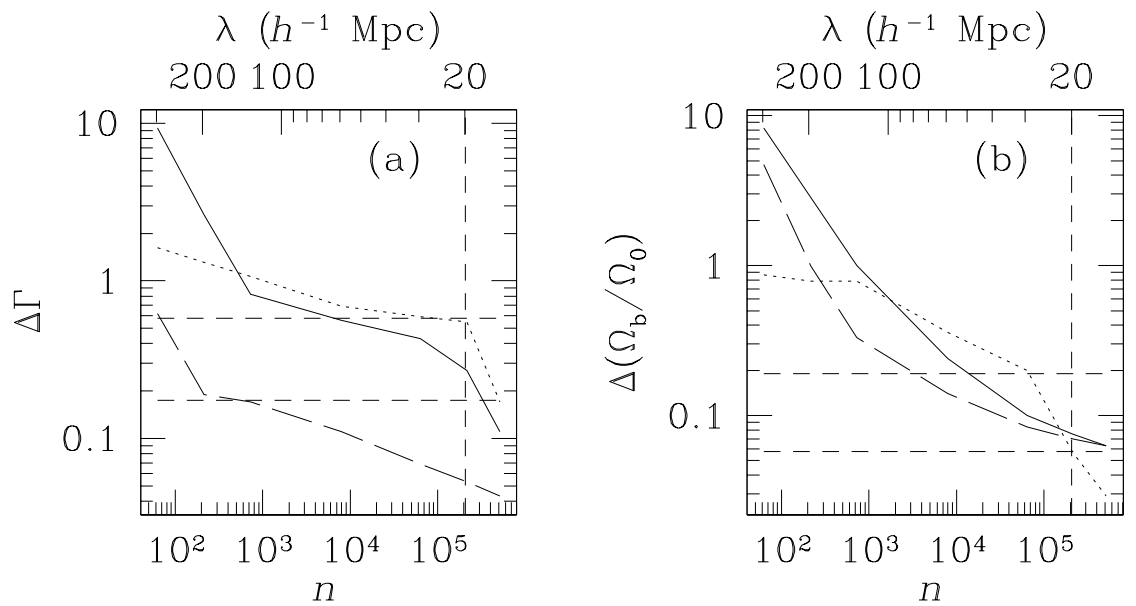

Fig. 7. - The computed uncertainties of (a) $\Gamma$ and (b) $\Omega_{b} / \Omega_{0}$. The solid line is Model 1, the dotted line is Model 2, and the long dashed line is Model 3. 

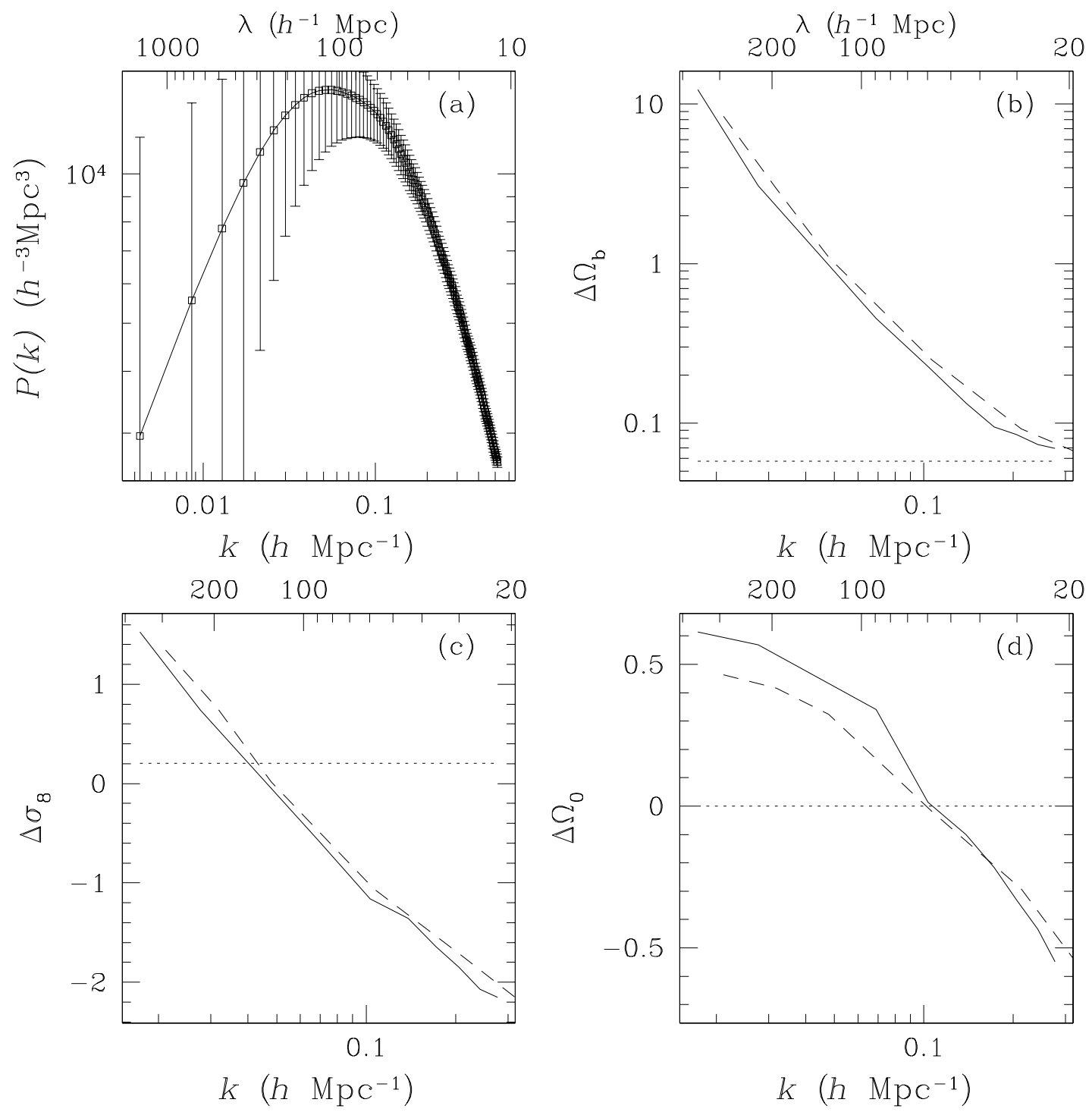

Fig. 8. - The associated errors on the Model $1\left(\Omega_{0}=1, \Omega_{b}=0.057\right)$ power spectrum and its parameters. (a) The errors on the banded power spectrum using the FKP error bars. (b) The uncertainty on $\Omega_{b}$ using FKP errors (solid) compared with the uncertainty of $\Omega_{b}$ computed using the covariance matrix (dashed), as a function of $|k|$. (c) The errors for $\sigma_{8}$. (d) The errors for $\Omega_{0}$. 